\title{
Quantification of operating reserves with high penetration of wind power considering extreme values
}

\author{
Johan S. Obando, Gabriel González, Ricardo Moreno \\ Energy and Mechanical Department, Universidad Autónoma de Occidente, Cali, Colombia
}

\begin{tabular}{l}
\hline \hline Article Info \\
\hline Article history: \\
Received May 9, 2019 \\
Revised Oct 28, 2019 \\
Accepted Nov 4, 2019 \\
\hline Keywords: \\
Extreme values \\
Montecarlo simulation \\
Operating reserves \\
Optimal power flow \\
Wind power \\
\hline
\end{tabular}

\begin{abstract}
The high integration of wind energy in power systems requires operating reserves to ensure the reliability and security in the operation. The intermittency and volatility in wind power sets a challenge for day-ahead dispatching in order to schedule generation resources. Therefore, the quantification of operating reserves is addressed in this paper using extreme values through Monte-Carlo simulations. The uncertainty in wind power forecasting is captured by a generalized extreme value distribution to generate scenarios. The day-ahead dispatching model is formulated as a mixed-integer linear quadratic problem including ramping constraints. This approach is tested in the IEEE-118 bus test system including integration of wind power in the system. The results represent the range of values for operating reserves in day-ahead dispatching.
\end{abstract}

Copyright $\odot 2020$ Institute of Advanced Engineering and Science. All rights reserved.

\section{Corresponding Author:}

Ricardo Moreno,

Energy and Mechanical Department,

Universidad Autónoma de Occidente,

Calle 25 \# 115-85. Cali-Colombia.

Email: rmoreno@uao.edu.co

\section{INTRODUCTION}

The high penetration of wind power into the power systems represents major challenges for operation and planning. In particular, in power system operation the deviation between expected and real power production is compensated by operating reserves. Given the wind power intermittency, the operating reserves are crucial to cover up the sudden changes in electricity production, therefore, the operating reserves must be available and enough in quantity to be dispatched in real time. The operating reserves are quantified usually day-ahead, sometimes intra-day ahead, to guarantee the security and operational reliability.

Wind forecasting errors bring great uncertainty to the systems operations, since the real-time wind power output may be very different from what is forecasted previously. The reliability of the system may be hampered in case of unforeseen decreases in wind power because the available ramping capability of on-line units in the system may not be sufficient to accommodate this change. For instance, a large upward ramp in wind power may be unfavorable in a system in which sufficient downward reserves from other resources are not available. Sometimes, during low load periods some units generates power according to ramp up and ramp down restrictions, in those cases, with low demand and high potential of wind power, wind generation may be spilled to maintain a state of operation with units already generating.

Therefore, the challenge is on the quantification of operating reserves under wind power uncertainty and intermittency on a day-ahead basis. The operating reserves should be enough to cope up extreme values of wind power generation. Given the importance about the integration of renewable resources, and particularly, wind power, this issue has been addressed in research with practical interest. The models includes the unit commitment formulation to integrate wind power along with thermal power, for instance, in [1] the authors propose a bioinspired algorithm to solve the problem given that the problem is nonlinear. Common formulations of day-ahead dispatching have been extended to account for the intermittent 
characteristics in the wind power [2-6]. A probabilistic approach has been addressed to capture the intermittency in day-ahead dispatching models. In the unit-commitment formulation, a stochastic approach is used, particularly, the authors in [7] report about the use of scenarios for generation with distribution probability functions, the demand is considered constant. Formulations with a stochastic approach has been suggested to manage wind power uncertainty in power system operation [8-11] and in microgrids in [12]. The flexibility in power system operation will be a critical issue under high penetration of wind power to deal with uncertainty and intermittency. In [10], the authors proposed an approach based on a model to clear a network-constrained electricity market with offers made by wind generators. In [13] the optimal power flow approach is addressed to minimize the rescheduling including total congestion cost minimization. In [14] the authors propose a dispatching model to consider simultaneously wind power and demand response.

The quantification of reserves under high penetration of wind power has been addressed in various electricity markets with different approaches, for instance, PJM (Pennsylvania, New Jersey and Maryland) in US classifies ancillary services as regulating and load-following reserves [15][13]. The operating reserves deals with the variability and intermittency of power wind from a power dispatching point of view, in fact, in [16], the authors affirm about the operational problems associated with renewable sources. In [17] an optimal reactive power scheduling problem is solved using an evolutionary search algorithm.

This paper provides a methodology to identify the proper level of reserves in power systems with penetration of wind power using extreme value theory as a novelty to characterize low probability cases with high impact in the operation reliability. This paper deals with the uncertainty and variability introduced by wind power to determine reserves in day-ahead dispatching. The model proposed in this paper is based on a model for day-ahead dispatching as a mixed-integer linear quadratic optimization problem with unit commitment. The model takes into account startup and shutdown characteristics for thermal units. The quantification of operating reserves is addressed via generalized extreme value functions.

We propose a quantification of ramp reserves in day ahead dispatching considering extreme values to characterize those high-impact low probability events. Therefore, in day-ahead dispatching or even in intra-day dispatching enough reserves has to be planned to compensate wind power volatility. The dispatching decision has to deal with the inherent uncertainty in order to dispatch economically the generation resources considering the system reliability at the same time. We propose the construction of wind power ramps trajectories through Monte-Carlo simulations to quantify the probability distribution of ramp reserves. Specifically, we propose a GEV (generalized extreme value) distribution function to produce wind power ramps trajectories considering $5 \%$ of the events are in the tails.

\section{MODELLING UNCERTAINTY WITH SCENARIOS}

In this section, we present the methodology and discussion about the generation of scenarios considering the uncertainty of wind power. Specifically, the generation of wind power trajectories for a 24-hour period to capture the uncertainty associated to wind power ramps. The use of GEV functions to generate trajectories capture low probability events as the intermittents power ramp given by wind power. The identification of ramps events are important to quantify reserves to compensate wind power ramps during the operation.

The most critical situations arise with extreme values of wind power ramps. Recent studies [18] has demonstrated that real-world wind power ramps exhibit heavier tails. In particular, the historical data from wind power generation in ERCOT (Electric Reliability Council of Texas) reveals that less than 5\% of hourly wind power ramps had a magnitude greater than three standard deviations [19], [20]. Those extreme values in the tails will require operating reserves during operation to compensate the deviations and to guarantee wind power integration. To quantify operating reserves considering the uncertainty associated to wind power ramps, we use extreme value theory to generate wind power trajectories with heavier tails. The wind power ramps can be suitable modeled by a generalized extreme value distribution (GEV) function given by,

$$
\Lambda(r)=\exp \left\{-\left[1+\varepsilon\left(\frac{r-\mu}{\beta}\right)\right]^{1 / \varepsilon}\right\}
$$

Defined on the set,

$$
r: 1+\varepsilon(r-u) / \beta>0
$$


Where the parameters satisfy,

$$
-\infty<\mu<\infty,-\infty<\varepsilon<\infty
$$

The model has three parameters: $\mu$ is a location parameter, $\beta$ is a scale parameter and $\varepsilon$ is a shape parameter. According to the parameter $\varepsilon$, there are three classes of distributions widely known as the Gumbel, Fréchet and Weibull families, or type I, II and III, respectively [21]. Specifically, if $\varepsilon=0$ then the distribution is a type I function, if $\varepsilon>0$ then the distribution is a type II function, and if $\varepsilon<0$ then the distribution is a type III function. The parameter $\varepsilon$ indicates the tail behavior, in other words, the distribution of wind power ramp events behind a threshold value given by $\mu$. In particular, the tail behavior for wind power ramp is given by type II distribution. In order to appreciate the tails in the GEV, Figure 1 shows a contrast of a Gaussian distribution and a GEV distribution.

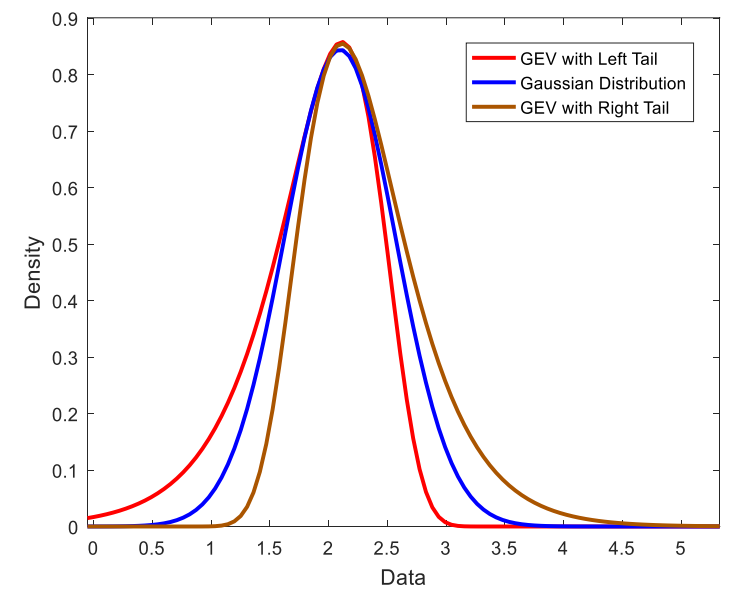

Figure 1. Gaussian distribution and GEV distribution

We use a Monte-Carlo framework to generate trajectories for a 24-hour period under the assumption than $5 \%$ of hourly wind power ramps had a magnitude greater than three standard deviations. Figure 2 and Figure 3, represents the wind power trajectories around a mean value to represent extreme values in the left and right tail respectively. The mean value corresponds to bid made by the wind power producer.

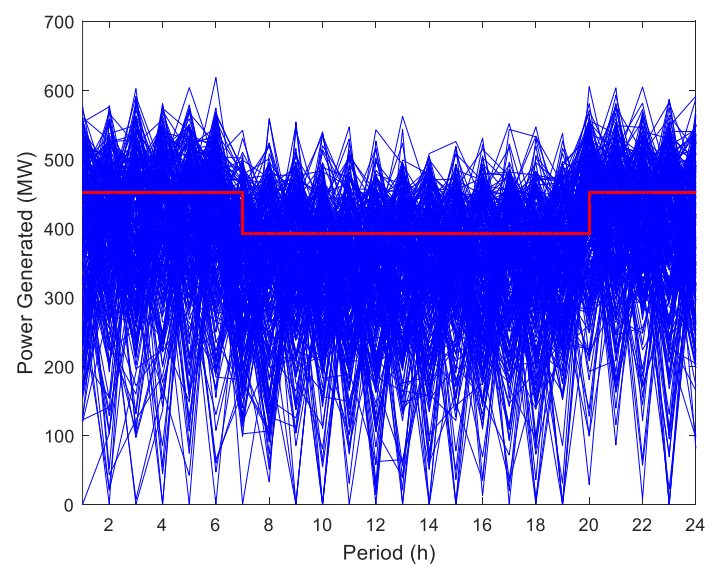

Figure 2. Wind power scenarios with values in the left tail

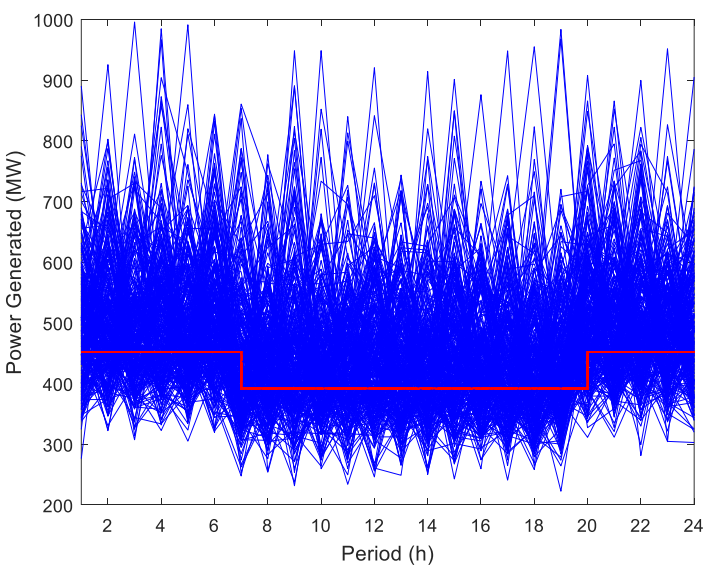

Figure 3. Wind power scenarios with values in the right tail 
For GEV distribution, extreme quantiles of the annual maximum distribution are given by the inverse of (1),

$$
z_{p}=\mu-\frac{\beta}{\varepsilon}\left[1-\{\log (1-p)\}^{-\varepsilon}\right]
$$

The $p$ quantile implies that with $(1-p)$ confidence wind power ramp is no greater than $z_{p}$. More precisely, the level $z_{p}$ is expected to be exceed on average once every $1 / p$ years.

\section{DAY-AHEAD DISPATCHING FORMULATION}

$\begin{array}{ll}C_{P}^{i, t}(\cdot) & \text { Production cost function for unit } i \text { at time } t \\ P^{i, t} & \text { Generation of unit } i \text { at time } \\ P^{w, t} & \text { Wind power forecast for unit } w \text { at time } t \\ P_{D}^{t} & \text { Power demand at time } t \\ P_{L}^{t} & \text { Power losses at time } t \\ T & \text { Number of periods in the planning horizon } \\ G & \text { Number of generating power units } \\ W & \text { Number of wind power units } \\ C_{\delta \pm}^{i, t}(\cdot) & \text { Cost of load-following for unit } i \text { at time } t \\ \delta_{ \pm}^{i, t} & \text { Load-following ramping for unit } i \text { at time } t \\ \delta_{\text {limit }}^{i} & \text { Load-following ramping limits for unit } i \\ P_{\min }^{i, t} & \text { Lower limit of power generation of unit unit } i \\ P_{\max }^{i, t} & \text { Upper limit of power generation of unit unit } i \\ u^{i, t} & \text { Binary commitment state for unit } i \text { at time } t \\ C_{u p}^{i, t} & \text { Startup cost for unit } i \text { at time } t \\ C_{d o w n}^{i, t} & \text { Shutdown cost for unit } i \text { at time } t \\ l_{i, t}^{i, t} & \text { Binary startup variable for unit } i \\ k^{i, t} & \text { Binary shutdown variable for unit } i \\ t & \text { Index for time }\end{array}$

The day-ahead dispatching is formulated as a mixed-integer linear quadratic optimization problem. The objective function is composed of production cost of power generation, cost for load-following (up and down), and costs for startup and shutdown of each generating unit (5). The operating costs of wind units are zero. The constraints includes the power balance constraint (7). The load following ramping limits are given by the restriction (8). The integer constraints indicating the binary startup and shutdown states are in (9). The power unit generating limits in (10). The constraint about the capacity limits, the tap changing and phase shifting for transformers are given in (11).

$$
\begin{aligned}
& \min \sum_{t \in T} \sum_{i \in G} C_{P}^{i, t}\left(P^{i, t}\right)+C_{\delta \pm}^{i, t}\left(\delta_{ \pm}^{i, t}\right)+b \\
& b=C_{u p}^{i, t} \cdot l^{i, t}+C_{d o w n}^{i, t} \cdot k^{i, t}
\end{aligned}
$$

Subject to:

$$
\begin{aligned}
& \sum_{i \in G} P^{i, t}+\sum_{w \in W} P^{w, t}=P_{D}^{t}+P_{\mathrm{L}}^{t} \\
& 0 \leq \delta_{ \pm}^{i, t} \leq \delta_{\text {limit }}^{i} \\
& l^{i, t} \in\{0,1\}, k^{i, t} \in\{0,1\}
\end{aligned}
$$




$$
\begin{aligned}
& P_{\text {min }}^{i, t} \leq P^{i, t} \leq P_{\text {max }}^{i, t} \\
& H\left(P^{i, t}, P_{D}^{t}, P_{\mathrm{L}}^{t}\right) \leq 0
\end{aligned}
$$

\section{CASE OF STUDY}

We propose to quantify the operating reserve in day-ahead dispatching performing an optimal power flow for the wind power trajectories generated with GEV functions according to the premise: than $5 \%$ of hourly wind power ramps had a magnitude greater than three standard deviations. In particular, we use the IEEE 39-bus test system, this system includes 10 generators, 46 branches and 19 loads. The data of the generators is listed in Table 1. The system load curve for a 24-hour period has a peak load of $4531 \mathrm{MW}$ at hour 20 as shown in Figure 4.

Table 1. Generator Data for the IEEE 39 Bus system

\begin{tabular}{ccccccc}
\hline Gen \# & $C_{\delta+}$ & $C_{\delta-}$ & $C_{v}$ & $C_{w}$ & $P_{\min }$ & $P_{\max }$ \\
\hline 1 & 6.9 & 6.9 & 920 & 736 & 0 & 250 \\
2 & 6.9 & 6.9 & 920 & 736 & 0 & 678 \\
3 & 6.9 & 6.9 & 920 & 736 & 0 & 650 \\
4 & 6.9 & 6.9 & 920 & 736 & 0 & 632 \\
5 & 6.9 & 6.9 & 920 & 736 & 0 & 508 \\
6 & 6.9 & 6.9 & 920 & 736 & 0 & 650 \\
7 & 6.9 & 6.9 & 920 & 736 & 0 & 560 \\
8 & 6.9 & 6.9 & 920 & 736 & 0 & 540 \\
9 & 6.9 & 6.9 & 920 & 736 & 0 & 830 \\
10 & 6.9 & 6.9 & 920 & 736 & 0 & 1000 \\
\hline
\end{tabular}

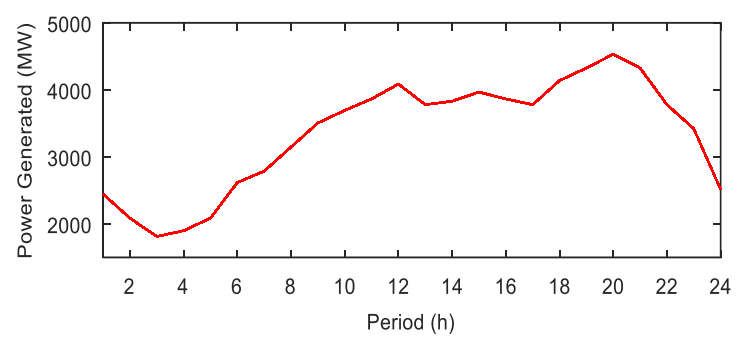

Figure 4. System load curve

The cost data are quadratic functions as reported in [22-24]. Table 2 lists the cost functions for the ten conventional generators. This quadratic cost functions characterize more appropriately the cost structure of thermal generation in power systems.

Table 2. Cost Functions

\begin{tabular}{cl}
\hline Gen & \multicolumn{1}{c}{ Cost Function $[\$]$} \\
\hline 1 & $C_{1}=0.00194 P^{2}+7.85 P+310$ \\
2 & $C_{2}=0.0035 P^{2}+8.5 P+260$ \\
3 & $C_{3}=0.00482 P^{2}+7 P+78$ \\
4 & $C_{4}=0.00128 P^{2}+6.4 P+459$ \\
5 & $C_{5}=0.0024 P^{2}+6 P+80$ \\
6 & $C_{6}=0.0032 P^{2}+5.8 P+400$ \\
7 & $C_{7}=0.0053 P^{2}+6.24 P+120$ \\
8 & $C_{8}=0.00185 P^{2}+8.4 P+60$ \\
9 & $C_{9}=0.0025 P^{2}+5.75 P+450$ \\
10 & $C_{10}=0.00142 P^{2}+8.2 P+510$ \\
\hline
\end{tabular}

Quantification of operating reserves with high penetration of wind power considering.. (Johan S. Obando) 


\section{SIMULATION RESULTS}

In this section, we provide simulation results to quantify operating reserves with high penetration of wind power. The day-ahead dispatching model for the IEEE-39 bus test system is a mixed-integer linear quadratic optimization problem. We use GUROBI 7.5.1 [25] under the platform of Matpower [26] as solver. The simulations were completed by a PC with Intel Core i7 - 3537U CPU @ 2.00 GHZ with 8.00 GB RAM. We run a day-ahead dispatching to obtain results from scenarios to capture wind power uncertainty. We run the wind power scenarios for both tails. The cost function, in Figure 5, reveals values in the right tail given the wind power modelling with generalized extreme values, corresponding to scenarios with high operating costs. The parameters for the generalized extreme function are $\varepsilon=-0.346, \beta=4041.57, \mu=513638$.

Figure 6 reveals values in the left tail, corresponding to scenarios with high penetration of wind power. The generalized extreme values parameters are $\varepsilon=-0.224, \beta=4821.49, \mu=528257$, it represents the cost function in terms of a GEV function.

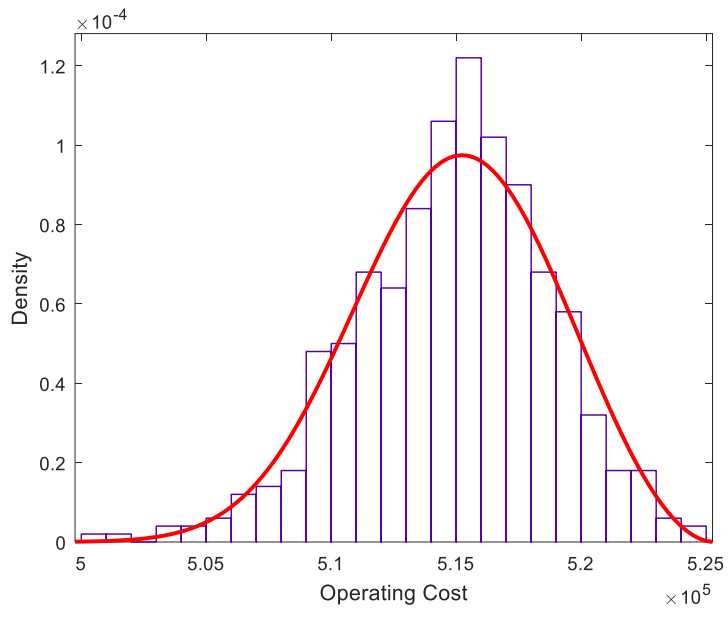

Figure 5. Operating cost for the IEEE 39-bus test system with values in the right tail

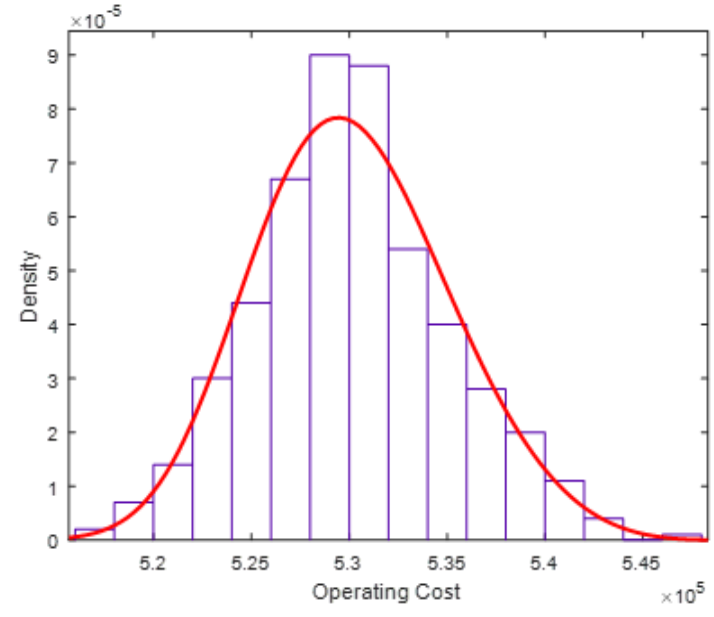

Figure 6. Operating cost for the IEEE 39-bus test system with values in the left tail

For wind power, the percentage of cases beyond three sigmas for each period is reported in Table 3. In hour two, there is a five percentage $(5 \%)$ of cases where the wind power output is beyond three sigmas in the right tail. Wind generation is a variable energy resource with changing availability level over the time (variability), which cannot be predicted with perfect accuracy (uncertainty) [27] As wind power increases, the additional variability and uncertainty introduced in the system will cause an increase of operating reserves in the system [19]. In order to proposed a operaing reserve function in MW related to the power system, we quantified the operating reserve up in the left stage and the operating reserve down in the right stage.

Table 3. Percentaje of cases in each hour beyond three sigmas

\begin{tabular}{cccccc}
\hline Period [Hour] & Cases $(\%)$ & Period [Hour] & Cases $(\%)$ & Period [Hour] & Cases $(\%)$ \\
\hline 1 & 4.2 & 9 & 4.6 & 17 & 1.6 \\
2 & 5 & 10 & 1.4 & 18 & 2.6 \\
3 & 3.4 & 11 & 2.6 & 19 & 1.8 \\
4 & 4 & 12 & 2.6 & 20 & 2.8 \\
5 & 3.2 & 13 & 2.2 & 22 & 3.2 \\
6 & 4.2 & 14 & 2 & 22 & 3.4 \\
7 & 4 & 15 & 2.8 & 23 & 1.6 \\
8 & 2.2 & 16 & 1.8 & 24 & 2 \\
\hline
\end{tabular}

In addition, we plot downward ramping reserves and upward ramping reserves under the modelling of wind power using generalized extreme values to quantify reserves. Figure 7 shows the downward ramping reserves, the mean value is $2600 \mathrm{MW}$, but, the key result is about the values in the right tail. We observe values around $3100 \mathrm{MW}$. This values indicates that the operation planning in day-ahead dispatching may 
require $3100 \mathrm{MW}$ to mitigate the wind power uncertainty. Figure 8 shows the upward ramping reserves. In both cases, there is values in the tails. The operating reserves are adjusted to a GEV function type II, the parameters of scale, location and shape are indicated in Table 4 for downward and upward operating reserves.

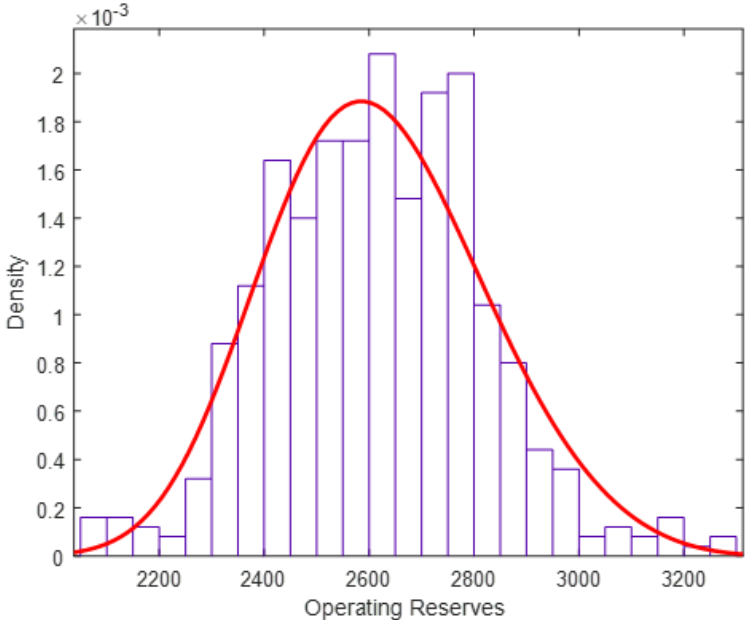

Figure 7. Downward ramping reserve quantities for the right tail in the IEEE 39-bus test system

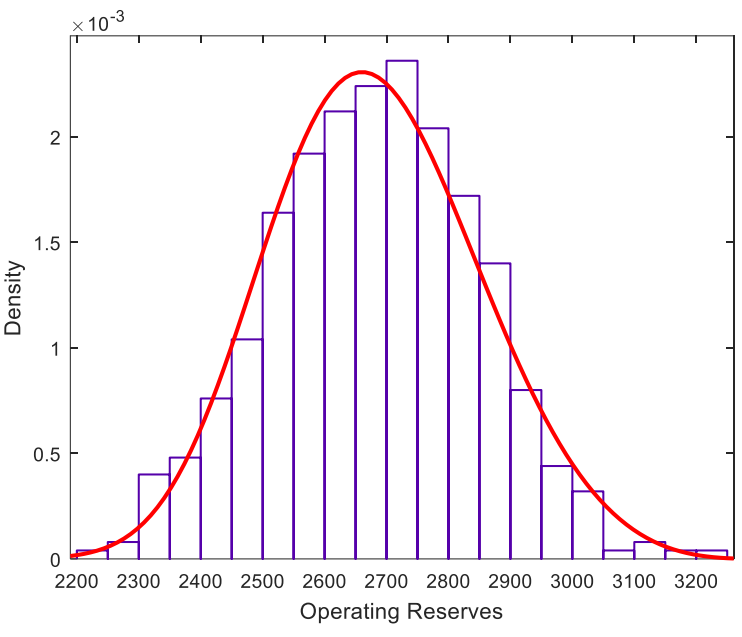

Figure 8. Upward ramping reserve quantities for the left tail in the IEEE 39-bus test system

Table 4. Operating reserves function

\begin{tabular}{cccc}
\hline & $\varepsilon$ & $\beta$ & $\mu$ \\
\hline Left Tail & -0.215 & 200.1 & 2537.5 \\
Right Tail & -0.224 & 164.3 & 2617.5 \\
\hline
\end{tabular}

\section{CONCLUSION}

This paper provides insights about the dispersion related with wind power and an aproach to quantify the reserves needed to mitigate the intermittency. This paper proposes a mixed integer linear quadractic problem to characterize day-ahead dispatching. The results offers evidences that wind power uncertainty have great impact on the scheduling of generating units in the day-ahead market with implications on ramping reserves. The operating reserves follow a generalized extreme value (GEV) distribution if the wind power follow a GEV function type II as its suggest by the analysis of wind data of wind plants. The approach proposed in this paper allows to deduce the expected distribution function for operating reserves.

\section{ACKNOWLEDGEMENTS}

The authors acknowledge the support of the Universidad Autónoma de Occidente in Cali, Colombia.

\section{REFERENCES}

[1] S. S. Sakthi, R.K. Santhi, N. M. Krishman, S. Ganesan, S. Subramanian, "Wind Integrated Thermal Unit Commitment Solution using Grey Wolf Optimizer," International Journal of Electrical and Computer Engineering (IJECE), vol. 7, no. 5, pp. 2309-2320, Oct. 2017.

[2] R. A. Jabr and B. C. Pal, "Intermittent wind generation in optimal power flow dispatching," IET Gener. Transm. Distrib, vol. 3, no. 1, pp. 66-74, Jan 2009. http://doi.org/10.1049/iet-gtd:20080273

[3] S. Reddy, "Multi-objetive based optimal energy and reactive power dispatch in deregulated electricity markets," International Journal of Electrical and Computer Engineering (IJECE), vol. 8, no. 5, pp. 3427-3435, Oct. 2018.

[4] H. Zhang and P. Li, "Probabilistic analysis for optimal power flow under uncertainty," IET Gener. Transm. Distrib, vol. 4, no. 5, pp. 553-561, May 2010. http://doi.org/10.1049/iet-gtd.2009.0374

[5] R. Entriken, A. Tuohy, and D. Brooks, "Stochastic optimal power flow in systems with wind power," USA, Jul. 2011, pp. 1-5. http://doi.org/10.1109/PES.2011.6039581 
[6] B. Banhthasit, C. Jamroen, S. Dechanupaprittha, "Optimal generation schedeluing of power system for maximum renewable energy harvesting and power losses minimization," International Journal of Electrical and Computer Engineering (IJECE), vol. 8, no. 4, pp. 1954-1966, Aug. 2018.

[7] C. S. Saunders, Point estimate method addressing correlated wind power for probabilistic optimal power flow, IEEE Trans. Power Syst., vol. 29, no. 3, pp. 1045-1054, May 2014. http://doi.org/ 10.1109/TPWRS.2013.2288701

[8] A. Papavasiliou and S. S. Oren, "Multiarea stochastic unit commitment for high wind penetration in a transmission constrained network," Oper. Res., vol. 61, no. 3, pp. 578-592, 2013.

[9] F. Bouffard and F. D. Galiana, "Stochastic security for operations planning with significant wind power generation," IEEE Trans. Power Syst.,vol. 23, no. 2, pp. 306-316, May 2008. http://doi.org/10.1109/TPWRS.2008.919318

[10] J. M. Morales, A. J. Conejo, and J. "Perez-Ruiz, Economic valuation of reserves in power systems with high penetration of wind power," IEEE Trans. Power Syst., vol. 24, no. 2, pp. 900-910, May 2009. http://doi.org/ 10.1109/TPWRS.2009.2016598

[11] A. Dalabeeh, A. Almofleh, A. Alzyoud, H. Ayman, "Economical and reliable expansion alternative of composite power system under restructuring," International Journal of Electrical and Computer Engineering (IJECE), vol. 8, no. 6, pp. 4790-4799, Dec. 2018.

[12] T. Diep-Thanh, Q. Nguyen-Phung, H. Nguyen-Duc, "Stochastic control for optimal power flow in islanded microgrid," International Journal of Electrical and Computer Engineering (IJECE), vol. 9, no. 2, pp. 1045-1057, Apr. 2019.

[13] S. Kim, S. Reddy, "Optimal power flow based oncgestion management using enhanced genetic algorithms," International Journal of Electrical and Computer Engineering (IJECE), vol. 9, no. 2, pp. 875-883, Apr. 2019.

[14] R. Moreno, J. Obando, G. Gonzalez, "An integrated OPF dispatching model with wind power and demand response for day-ahead markets," International Journal of Electrical and Computer Engineering (IJECE), vol. 9, no. 4, pp. 2794-2802, Aug. 2019.

[15] E. Ela, B. Kirby, E. Lannoye, M. R. Milligan, D. Flynn, B. Zavadil, and M. O' Malley, "Evolution of Operating Reserve Determination in Wind Power Integration Studies, " in Proceedings of the IEEE Power and Energy Society General Meeting, Minneapolis, MN, USA, July 25-29, 2010. http://doi.org/ 10.1109/PES.2010.5589272

[16] A. N. Afandi, A. P. Wibawa, S. Padmantara, G. Fujita, W. Triyana, Y. Sulistyorini, H. Miyauchi, N. Tutkun, M. EL-Shimy Mahmoud, X. Z. Gao, "Designed Operating Approach of Economic Dispatch for Java Bali Power Grid Areas Considered Wind Energy and Pollutant Emission Optimized Using Thunderstorm Algorithm Based on Forward Cloud Charge Mechanism," International Review of Electrical Engineering (IREE), vol. 13 n. 1, February 2018, pp. 59-68. http://doi.org/10.15866/iree.v13i1.14687

[17] S. Surrender, "Optimal reactive power sceduling using cuckoo search algorithm," International Journal of Electrical and Computer Engineering (IJECE), vol. 7, no. 5, pp. 2349-2356, Oct. 2017.

[18] D. Ganger, J. Zhang, and V. Vittal, "Statistical characterization of wind power ramps via extreme value analysis," IEEE Trans. Power Syst., vol. 29, no. 6, pp. 3118-3119, Nov. 2014. http://doi.org/ 10.1109/TPWRS.2014.2315491

[19] Y. Wan, "Analysis of wind power ramping behavior in ERCOT," Nat. Renewable Energy Lab., Golden, CO, USA, Tech. Rep. TP-5500-49218, Mar. 2011.

[20] Zhao, Jie, et al. "Quantifying risk of wind power ramps in ERCOT," IEEE Transactions on Power Systems 32.6 (2017): 4970-4971. http://doi.org/10.1109/TPWRS.2017.2678761

[21] J. Pickands, III, "Statistical inference using extreme order statistics," Ann. Statist., vol. 3, pp. 119-131, 1975.

[22] R. Zárate-Miñano, F. Milano and A. J. Conejo, “An OPF Methodology to Ensure Small-Signal Stability,” IEEE Trans. Power System, vol. 26, no. 3, Aug. 2011. http://doi.org/ 10.1109/TPWRS.2010.2076838

[23] T. Dai, W. Qiao and L. Qu, "Real-time Optimal Participation of Wind Power in an Electricity Market," in IEEE Innovative Smart Grid Technologies Conf., Tianjin, China, 2012.

[24] S. Jang, H. Jung, J. Park, and S. King, "A new network partition method using the sensitive of marginal cost under network congestion," IEEE Power Engineering Society Summer Meeting, 2001. http://doi.org/10.1109/PESS.2001.970326

[25] The GUROBI Manual. Accessed on May 5, 2017. [Online]. Available: https://www.gurobi.com/documentation/7.5/refman/index.html.

[26] Matpower Optimal Scheduling Tool (MOST) package. Accessed on Apr. 3, 2017. [Online]. Available: http://www.pserc.cornell.edu/ matpower/manual.pdf

[27] Black, M., Strbac, G. Value of bulk energy storage for managing wind power fluctuations, IEEE Trans. Energy Convers., 2007, 22, (1), pp. 197-205. http://doi.org/10.1109/TEC.2006.889619 